\title{
Real-Time Channel Sounding for Channel-Adaptive Data Links
}

\author{
Alexander R. Ganis ${ }^{1}$, Christian Bluemm ${ }^{1}$, Christoph Heller ${ }^{1}$ and Mirko Loghi ${ }^{2}$ \\ ${ }^{1}$ Airbus Group, Munich, Germany, \\ AlexanderRudolf.Ganis@eads.net \\ ${ }^{2}$ University of Udine, Udine, Italy
}

\begin{abstract}
:
The development of wideband mobile communications systems requires a good knowledge of the characteristics of the mobile channel as described by its impulse response. Such impulse response can be modeled as a tapped delay line with delays, amplitudes and phases associated with each tap. In order to obtain satisfactory estimates of these parameters and to determine delay spread, Doppler spread and the channel scattering function, a real-time channel sounder is required. This paper focuses on the hardware implementation of a real-time channel sounder, on an Innovative-DSP X6400M Software-Defined-Radio platform based on a Xilinx Virtex-6 FPGA. The real-time channel sounder has been developed in VHDL and implemented with Xilinx ISE AND Xilinx System Generator design, simulation and analysis softwares. In order to visualize the measured channel scattering function, a graphical user interface has been created. Testing of the channel sounder using a channel emulator allowed to verify the proper operation of the project.
\end{abstract}

Key words: channel sounding, scattering function, Doppler shift, delay shift, and FPGA.

\section{Introduction}

The goal of channel measurements is to obtain a description of the channel transfer function, or conversely, the channel impulse response which describes the characteristics of the mobile channel which can be modeled as a tapped delay line (TDL) with delays, amplitudes and phases associated with each tap. In order to obtain satisfactory estimates of these parameters and to determine delay spread, Doppler spread and the channel scattering function, a real-time channel sounder is required. On this basis, an easy-to-use channel sounding technique has been developed in order to measure the time-variant impulse response and the Doppler-variant impulse response. In order to detect the time-variant impulse response $h(\tau)$, the proposed sounding technique works with frequency modulated continuous wave (FMCW) signal (chirps), which exhibit a linear frequency decrease over time. More precisely, the technique relies on the transmission of a periodically repeated complex chirp signal. Compared to other sounding methods, FMCW sounding provides good control of the spectrum of the test signal by selecting the start and stop frequency of the sweep. This ensures that the signal emissions do not violate the frequency range, if assigned, during the channel measurements. This paper focuses on the design and implementation of the described wireless channel sounding system on an Innovative-DSP X6-400M Software-Defined-Radio platform based on a Xilinx Virtex-6 FPGA [1]. The aim behind the implementation was to implement the channel sounder functions together with the communication functions on the same hardware platform. The decision was made to use a Field Programmable Gate Array (FPGA) based SDR (Software Defined Radio) platform, since it provides the required computing power and flexibility for this implementation.

The paper is structured as follows. Section II introduces the basics of channel modeling. Section III outlines the proposed channel sounding technique, section IV and V the setup and dimensioning that have been chosen. The measurement details and results are given in section $\mathrm{VI}$ and the conclusion is drawn in section VII.

\section{Background on channel modeling}

A wireless fading channel is most efficiently characterized as a linear time-variant system, which in turn is fully described with its impulse response. According to [2], the impulse response $h(t, \tau)$ at time $t$ to an impulse at time $t-\tau$ can be expressed as: 


$$
\begin{aligned}
h(t, \tau) & =\sum_{i=0}^{N-1} a_{i}(t, \tau) \cdot e^{j 2 \pi f_{0} \tau_{i}(t)+\phi_{i}(t, \tau)} \\
& \cdot \delta\left(\tau-\tau_{i}(t)\right)
\end{aligned}
$$

The expression reflects the incoherent superposition of N-MPCs with mutually independent variables, the path gain $a_{i} \in \mathbb{R}$ and the phase $\phi_{i} \in\left[0,2 \pi\left[\right.\right.$, with $\tau_{i}$ being the propagation delay of the MPC $i \in\{1 \ldots N\}$. The phase term $j 2 \pi f_{0} \tau_{i}(t)+\phi_{i}(t, \tau)$ represents the phase shift due to free space propagation of the MPC under the carrier frequency $f_{0}$, plus any additional phase shifts which result from the channel. Both main propagation mechanisms, the phase shift, e.g. due to the Doppler effect and the propagation delay of the MPCs are represented in the above equation. Propagation delay is the consequence of the limited propagation velocity of electromagnetic signals in air, which roughly equals the speed of light in vacuum, $c_{0}$. The path delay then is directly proportional to the path length $l$ the signal travels from transmitter antenna to receiver antenna. The relation between path length and delay can be seen as:

$$
\tau=\frac{l}{c_{0}}
$$

These signal copies sum up at the receiver and result in a so called delay spread. The maximum amount of delay spread equals the difference of the path delay of the longest path and the one of the shortest path. Due to the relative motion between transmitter, receiver or reflecting objects, another channel effect needs to be considered: each multipath wave undergoes a shift in frequency. This shift in the received signal frequency is referred to as the Doppler shift, and is directly proportional to the velocity of the mobile with respect to the direction of arrival of the received multipath signal. When the mutual distance between the wireless communication partners decreases, a positive frequency shift is introduced and when the distance increases, a negative frequency shift is introduced. Given a transmitter, which moves with the velocity $v$ in an angle $\varphi$ relative to the line of sight (LOS) path to the receiver, if the transmitted signal has a carrier frequency $f_{0}$, then the Doppler shift $f_{D}$, experienced as carrier frequency offset (CFO) by the received signal is given as:

$$
f_{D}=f_{0} \frac{v \cdot \cos (\varphi)}{c_{0}}
$$

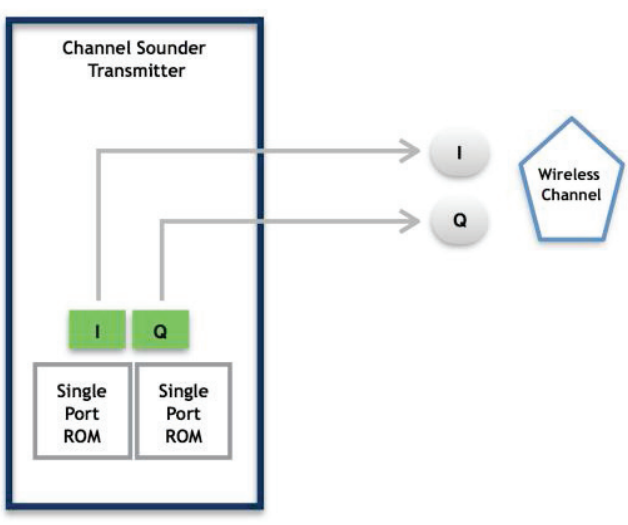

Fig. 1. Block Diagram of the Channel Sounder transmitter implemented in Xilinx System Generator.

\section{The proposed channel sounder}

In order to detect the time-variant impulse response $h(\tau)$ from equation (1), the proposed sounding technique works with frequency modulated continuous wave (FMCW) signal (chirps), which exhibit a linear frequency decrease over time. More precisely, the technique relies on the transmission of a periodically repeated complex chirp signal $s_{C}(t)$ [3]:

$$
s_{C}(t)=e^{j 2 \pi f_{0} t} \cdot e^{j 2 \pi\left(\frac{f_{C}}{2}-\frac{f_{C}}{T_{C}} t\right) t}, 0 \leq t \leq T_{C}
$$

The channel sounder excites the channel with the signal $s_{C}(t)$ defined in equation (4), whose (negative) frequency slope is given by $-f_{C} / T_{C}$. This means the chirp sweeps linearly from $-f_{C} / 2$ to $f_{C} / 2$ around the carrier frequency $f_{0}$ within the period $T_{C}$. The transmitter chirp signals are defined as I/Q components (generated off-line with MATLAB) and stored in a look-up-table, which is continuously read out from two ROMs, as illustrated in Figure 1 . In contrast to previously published techniques for chirp-based channel sounding $[4,5]$, the chirp signal is defined directly in baseband domain as inphase $(\mathrm{I})$ and quadrature $(\mathrm{Q})$ component. Since the baseband chirp is complex, with $Q$ being the $90^{\circ}$ phase-shifted version of I, single sideband modulation is achieved without further signal conditioning, such as bandpass filtering, which is a great benefit regarding linearity. After transmission over the air interface and downconversion into the baseband domain on the receiver side, the $\mathrm{I} / \mathrm{Q}$ signal is ready for demodulation. 

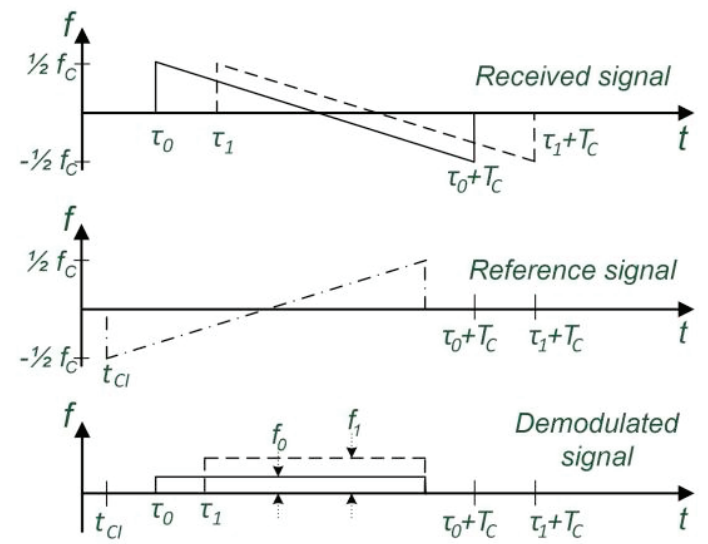

Fig. 2. Block Diagram of the Channel Sounder transmitter implemented in Xilinx System Generator.

This is done through matched filtering (frequency mixing) with an inverted complex chirp $s_{C I}(t)$ as reference signal, which starts at time $t_{C I}(t)[3]$ :

$s_{C}(t)=e^{j 2 \pi f_{0} t} \cdot e^{j 2 \pi\left(-\frac{f_{C}}{2}+\frac{f_{C}}{T_{C}} t\right) t}, t_{C I} \leq t \leq T_{C}+t_{C}$

Figure 2 illustrates the demodulation process with a simple two-path channel example. According to [3], a transmitted chirp signal will spread under a multipath channel into $N$ weighted and time-shifted chirp copies $y_{C}(t)$. The time span $\left[\tau_{1}, t_{C I}+T_{C L}\right]$ is considered, within which all received chirps overlap the reference signal $s_{C I}(t)$. Demodulation yields [3]:

$$
\begin{aligned}
u(t) & =s_{C I}(t) \cdot y_{C}(t) \\
& =s_{C I}(t) \cdot \sum_{i=0}^{N-1} a_{i} \cdot e^{-j \phi_{i}} \cdot s_{C}\left(t-\tau_{i}\right) \\
& =\sum_{i=0}^{N-1} a_{i} \cdot e^{-j \phi_{i}} \cdot e^{j 2 \pi f_{C} \tau_{i}\left(-\frac{\tau_{i}}{T_{C}}-\frac{1}{2}\right)} e^{j 2 \pi \frac{2 f_{C}}{T_{C}} \tau_{i} t}
\end{aligned}
$$

It turns out that $u(t)$ exhibits two independent components for each MPC, a phase component and a frequency component (phasor). Here, the key is that the phasor frequencies $f_{i}$, are directly proportional to the path delays $\tau_{i}$, with the factor $2 f_{C} / T_{C}$, which is two times the frequency slope:

$f_{i}=2 \frac{f_{C}}{t_{C}} \tau_{i}$

Thanks to this relation, the desired impulse response $h(\tau)$ is easily extracted after demodulation in two steps. First, an FFTtransformation (of size qFFT1) applied on the demodulated signal $u(\tau)$ extracts the phasor frequencies $f_{i}$ for each MPC. Second, applying equation (7) on the result yields the path delays $\tau_{i}$ for each MPC and thus the delay spread function $h(t, \tau)$ with an accuracy given by the sampling frequency $f_{s}$ and the FFT size qFFT1. Similarly to how the transmitter chirp signals are generated in the channel sounder's transmitter, the inverted complex chirp signals $s_{C I}(t)$, which are used for demodulation through matched filtering (frequency mixing), are previously generated with MATLAB in an I/Q format and stored in a look-up-table, continuously read out from a ROM Block, as illustrated in Figure 4.

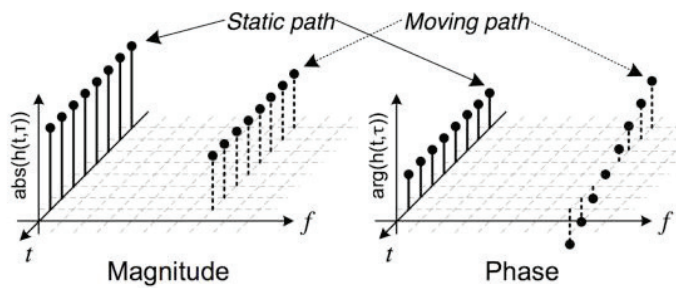

Fig. 3. The relation of time-variant impulse response and Doppler-variant impulse response.

The step from delay spread sounding to Doppler spread sounding is small. Another Fourier analysis is applied on multiple consecutive time-variant impulse responses $h(t, \tau)$. Figure 3 illustrates this relation in terms of the two-path channel example from Figure 2, assuming that the transmitter (TX) moves along the line-of-sight (LOS) path towards the receiver (RX). Depending on the actual frequency offset (e.g. due to Doppler), the individual multi-path component (MPC) rotates with measurement time. Each horizontal cut in Figure 3 stands for one individual delay spread measurement. In this simplied figure, the left path refers to a non-line-of-sigth (NLOS) MPC, having no frequency offset under this angle of motion. This turns the phase of this frequency component into a constant. By contrast, the right path refers to a LOS path and features a frequency offset that becomes visible in the phase rotation. The speed of phase rotation of each of qFFT1 available frequency components is directly proportional to the frequency offset on the corresponding MPC. Thus, applying another FFT of size qFFT2 on a set of delay spread functions in time-direction yields the frequency offset for an individual MPC. 


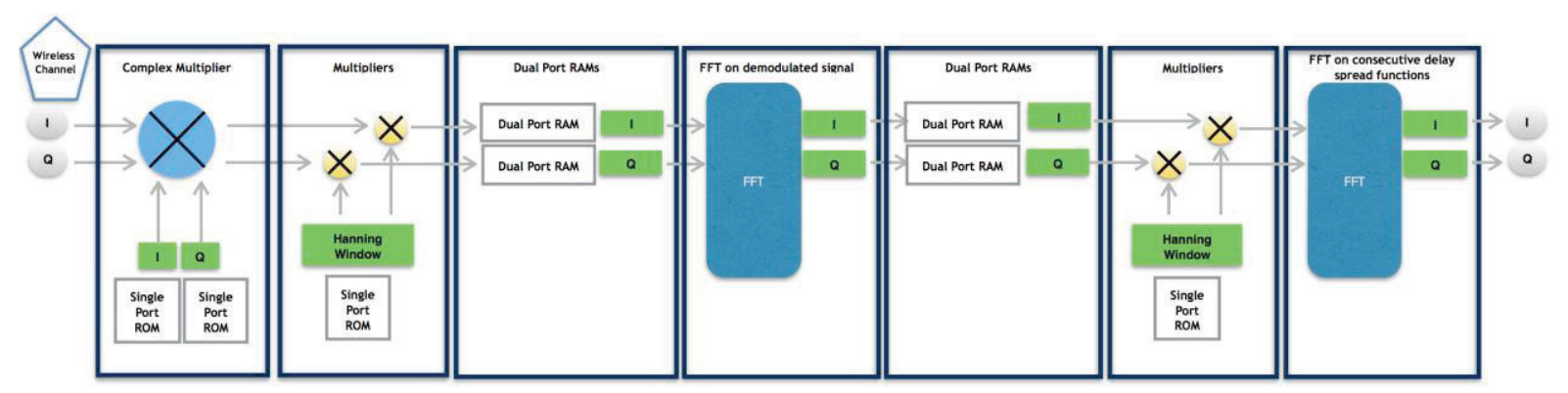

Fig. 4. Block Diagram of the Channel Sounder receiver implemented in Xilinx System Generator.

Doing so for all multi-path components results in the Doppler-variant impulse response or channel's scattering function. However, in order to do so, it is necessary to reorder the samples so that the second FFT operates the transformation on the correct blocks of nonsequential samples and one way to do so is to use Dual Port RAMs so that data can be written on port $A$ and read from port $B$ in a different oder, as seen in Figure 4. This approach further on increases the flexibility of the system as it enables us to apply digital processing on the signals before the FFT transformation is applied. This can be seen in Figure 4, where a Hanning filtering block is used in order to remove unwanted aliasing effects before the transformation occurs.

\section{Setup and measurements}

Figure 5 illustrates the setup used in the lab. Because of the graphical nature of Xilinx System Generator, the overall design can be viewed as a modular system and HDL code can be automatically generated ready to be processed in Xilinx ISE, without prior knowledge of HDL code itself. During the project development, several Xilinx Blocksets have been used, that contain functions for signal processing (e.g., FFTs), error correction, arithmetic, memories (e.g., FIFO, RAM, ROM), and digital logic. The channel sounder transmitted chirps generated in the transmitter part of the channel sounder are sent from the Innovative-DSP X6-400M Software-DefinedRadio platform to the input I and $Q$ ports of a Rohde \& Schwarz AMU200A channel emulator unit [6]. All fading parameters like the number of paths, delay, amplitude, and fading profile are here selected directly from the fading path table and applied to the chirps which will then be presented on the I and $Q$ output ports in the modulated form and sent back to the analog to digital converters (ADC) on the FPGA board. Thus, the received and modulated chirps will be processed in their $\mathrm{I}$ and $\mathrm{Q}$ format by the channel sounder's receiver, which after the digital signal processing and double FFT implementation, will send the processed samples to the top-level design where the samples will be stored in buffers and packed for Ethernet transmission to a laptop. The laptop is running a $\mathrm{C}++$ generated graphic user interface (GUI) that establishes a connection with the FPGA board, configures it and starts receiving and processing the sent samples in burst packets of 256 samples for 1024 times. The GUI stores each received block of data into a matrix that will be read by the plotting routine implemented in the $\mathrm{C}++$ as soon as the sounding process is completed. The channel's scattering function is plotted in the graphic user interface. The GUI is implemented in a way that it enables a user to decide to continuously trigger the transmitter and receiver parts of the channel sounder achieving a real-time channel sounding implementation. The result is a real-time plotting of the channel's scattering function in the GUI.

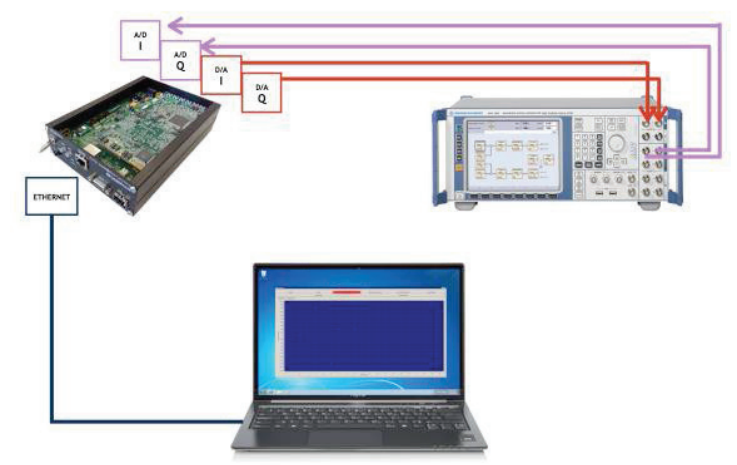

Fig. 5. Channel Sounder testing equipment.

\section{Dimensioning}

The proposed technique offers great flexibility for delay and Doppler spread sounding in terms of range and accuracy. The configurable parameters are listed in table 1 together with an adequate choice for the testing scenario and the available on board resources. The starting point for parameterization is the chirp bandwidth. For the purpose of folding, the channel sounder's sampling frequency was set to $f_{s}=f_{C}=50 \mathrm{MHz}$. To avoid aliasing problems, this sampling frequency is kept throughout the whole signal processing chain. Now, the required Doppler spread range for sounding is 
considered. The maximum Doppler spread range in a lab environment when using a

Tab. 1: Configurable parameters and chosen settings

\begin{tabular}{|c|c|}
\hline Parameter & Values \\
\hline $\begin{array}{c}\text { Sampling } \\
\text { frequency } f_{s}=f_{C}\end{array}$ & $50 \mathrm{MHz}$ \\
\hline Chirp duration TC & $\begin{array}{c}20.48 \mu \mathrm{s} \\
=1024 \text { samples }\end{array}$ \\
\hline Chirp pulses & 256 \\
\hline FFT size qFFT1 & 1024 \\
\hline FFT size qFFT2 & 256 \\
\hline
\end{tabular}

channel emulator is given by the Doppler frequency setting range of the device. In the case of the Rohde \& Schwarz AMU200A baseband signal generator and fading simulator used for testing, it's Doppler frequency range accessible via a fading path table is adjustable from $0 \mathrm{~Hz}$ to $1600 \mathrm{~Hz}$ for each path. Assuming a maximum Doppler shift of $\pm f_{D}= \pm 1.6 \mathrm{kHz}$, it has been decided to allow for some additional shifts, e.g. due to TX/RX oscillator frequency offsets and to choose a parameter which will end up in giving a multiple number for the chirp repetition, in sample. Thus the Doppler spread range was set to $\pm 2.03 \mathrm{kHz}$. This implies that the time-variant impulse responses $h(t, \tau)$ are captured with a frequency of at least $2 \times 2.03=$ $4.06 \mathrm{kHz}$, so that the FFT transformation across these functions can yield the Doppler components in the required range. With a 50 $\mathrm{MHz}$ sample rate, the chirp pulses thus have to be repeated at the latest every $50 \mathrm{MHz} / 4.06$ $\mathrm{kHz}=12288$ samples, which is a multiple number of qFFT1 $=1024$, which is the chosen chirp signal length. It was not possible to use 12288 as parameter for the maximum chirp length, because of memory resources constraints that will be addressed in future works. A Doppler resolution of $4.06 / 256=15.9 \mathrm{~Hz}$ is achieved by applying an FFT of size $q F F T 2=$ 256 on the time-variant impulse responses of 256 consecutive chirps. The delay spread resolution rises proportionally with increasing frequency slope $f_{C} / T_{C}$, since delay differences translate into bigger frequency differences. However, if the slope becomes too steep, the time-discrete chirp pulse will not reach each frequency value, resulting in quantization effects after FFT processing. With the chirp bandwidth being fixed to $f_{C}=50 \mathrm{MHz}$, the frequency slope is only configurable by the chirp duration $T_{C}$. At the same time, this chirp duration $T_{C}$ sets the maximum delay spread range, which turns into a trade-off between delay spread range and resolution. As anticipated, for increased accuracy, the paths are extracted using an FFT of size qFFT1 = 1024 without zero padding. Hanning filtering reduces the leakage effect and minimizes the visibility of the discontinuities at the beginning and end of the chirp sequences. The above mentioned maximum chirp length of 1024 samples results in a maximum chirp duration of $T_{C}=1024 / 50=20.48 \mu \mathrm{s}$. The chirp's duration together with the frequency slope set the upper bound for the frequency resolution to $f_{s} / 1024=$ $48.8 \mathrm{kHz}$, which translates into a delay resolution of $20 \mathrm{~ns}$. Table 2 summarizes the ranges and accuracies, which the configuration of table 1 yields.

Tab. 2: Ranges and accuracies with the configuration of table 1

\begin{tabular}{|c|c|}
\hline Performance & Values \\
\hline $\begin{array}{c}\text { Delay spread } \\
\text { range }\end{array}$ & $0 \ldots 20.48 \mu \mathrm{s}$ \\
\hline $\begin{array}{c}\text { Maximum delay } \\
\text { spread resolution }\end{array}$ & $20 \mathrm{~ns}$ \\
\hline $\begin{array}{c}\text { Doppler spread } \\
\text { range }\end{array}$ & $-2.03 \ldots 2.03 \mathrm{kHz}$ \\
\hline $\begin{array}{c}\text { Maximum Doppler } \\
\text { spread resolution }\end{array}$ & $15.9 \mathrm{~Hz}$ \\
\hline
\end{tabular}

A MATLAB script file has been created containing the parameters in the configuration table 1. This approach enables a flexible implementation of the channel sounder. Many parameters are easily editable like the direct and inverse chirp lengths and repetition, the channel sounding's sampling frequency, the FFT lengths, therefore making it possible to change the configuration of the channel sounder upon request.

\section{Results}

An exemple 5-path channel scenario has been created by setting up the path loss, delay and Doppler parameters for each path in the AMU200A channel emulator's path table, as illustrated in Figure 6. In this example, the delay shift is set to $1 \mu \mathrm{s}$ for path $1,3 \mu \mathrm{s}$ for path 2,6 $\mu$ s for path $3,7 \mu$ s for path 4 and $9 \mu$ s for path 5. The Doppler shift is set to $0 \mathrm{~Hz}$ for path 1, $+1600 \mathrm{~Hz}$ for path 2, -1600 Hz for path 3, 400 $\mathrm{Hz}$ for path 4 and $400 \mathrm{~Hz}$ for path 5 . Figure 7 shows a snapshot of the channel's scattering function generated by the channel sounder hardware implementation and visualized in the GUI's Visualization tab. The captured snapshot shows that there are 5 visible paths, each with different delay and Doppler shifts at the 
coordinates that were set in the path table in Figure 6 . The end result is that the proposed channel sounder accurately processed the

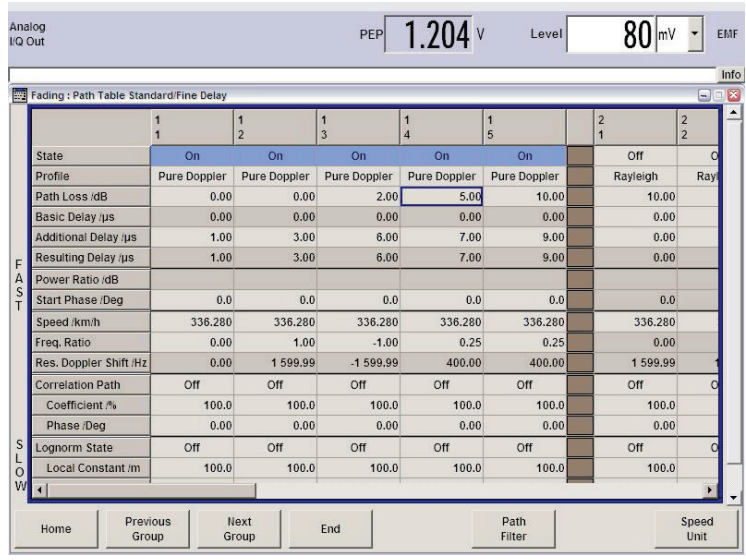

Fig. 6. Exemplary 5-path channel scenario.

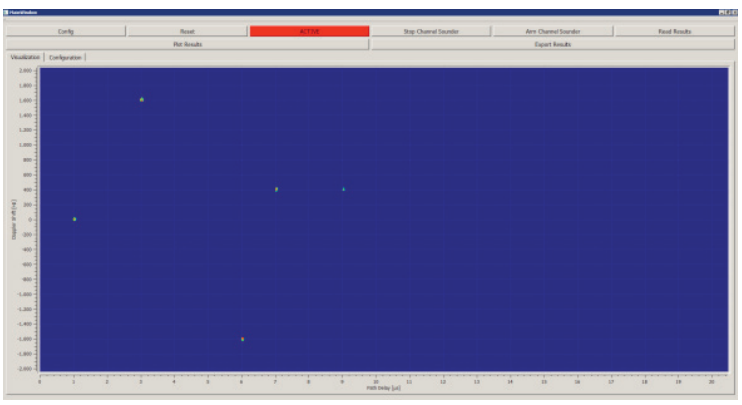

Fig. 7. Scattering function of the exemplary 5-path channel scenario (GUI).

delay and Doppler shift of each path, correctly presenting the channel scattering function of the emulated channel for each and every configuration that is set in the channel emulator.

\section{Conclusion and outlook}

The easy to use hardware implemented realtime channel sounder's benefits are that it offers great flexibility in terms of carrier frequency, instantaneous bandwidth, delay spread accuracy and Doppler spread accuracy. Furthermore, it is a low cost, flexibile solution and can be integrated in a complete system in order to achieve realistic channel modeling, based on channel sounding results, like it could be the case, but not limited to, an OFDM based waveform system. It is an easily implementable solution that can be combined with commercial off-the-shelf front-end hardware modules. The channel sounding results are directly applicable for tapped-delay line (TDL) modeling, simply by taking the delay, the Doppler shift and the normalized gain of each multi-path component as coefficients, instead of using stochastic random numbers with the advantage of having a realistic site-specific modeling with great accuracy and reliability, assuming a large number of simulations with a large number of channel sounding results.

Because it was implemented by taking into account flexibility since the early stages of the project, the proposed channel sounder offers great opportunities for improvement. The next step will be the integration of the proposed channel sounder in the complete proprietarily developed Orthogonal Frequency Division Multiplex (OFDM) based waveform, explained in detail in [7], in order to achieve adaptive remodulation of the system characteristics. In order to parameterize the OFDM link adequately, the propagation environment must be determined in terms of delay spread and Doppler spread, information combined in the so-called scattering function, which can be captured by the channel sounder.

Last but not least, testing of the channel sounder using field measurements with real channels will be made in the near future.

\section{References}

[1] Innovative Integration, "X6-400M PMC/XCM Module with Two 400/500 MSPS A/Ds, Two 500MSPS DACs, Virtex6 FPGA, 4 GB Memory and PCI/PCle", Datasheet; URL: http://www.innovativedsp.com/products.php?product $=X 6-400 M$ (2011).

[2] T. S. Rappaport, Wireless Communications: Principles and Practice, 2 ed. Prentice Hall PTR (2002).

[3] C. Bluemm, C. Heller, B. Fourestie and R. Weigel, Air-to-Ground Channel Characterization for OFDM Communication in C-Band, Signal Processing and Communication Systems (ICSPCS), 7th International Conference on, 1-8 (2013).

[4] S. Salous, Radio Propagation Measurement and Channel Modeling, Wiley (2013).

[5] S. Salous, Chirp Sounder Measurements for Broadband Wireless Networks and Cognitive Radio, Proc. 7th Int. Commun. Systems Networks and Digital Signal Process. (CSNDSP) Symp, 846-851 (2010).

[6] Rohde \& Schwarz, "AMU200A Baseband Signal Generator and Fading Simulator", Datasheet; URL: http://www.rohdeschwarz.us/file/AMU200A specs en.pdf (2007).

[7] C. Bluemm, C. Heller, and R. Weigel, SDR OFDM Waveform Design for a UGV/UAV Communication Scenario, Springer Journal of Signal Processing Systems 69, 11-21 (2012). 